\title{
Double-diffusive cellular fingering in the horizontally propagating fronts of the chlorite-tetrathionate reaction
}

\author{
Tamás Rica, Éva Pópity-Tóth, Dezső Horváth, Ágota Tóth* \\ Department of Physical Chemistry, University of Szeged, Rerrich Béla tér 1., Szeged, \\ H-6720, Hungary
}

\begin{abstract}
Horizontally propagating chemical fronts have been studied in the acidcatalyzed chlorite-tetrathionate reaction. Unusual cellular fingers develop in a relatively thin solution layer. The origin of pattern formation is a doublediffusive convection arising from the addition of a polyelectrolyte which immobilizes and reversibly removes the autocatalyst hydrogen ion in a controlled manner and drastically slows down the front. Convection therefore becomes significant in the thin horizontal solution layer. To corroborate our results we have measured the solution densities and viscosities, and determined the diffusion coefficients by PFGSE ${ }^{1} \mathrm{H}-\mathrm{NMR}$.

Key words: pattern formation, hydrodynamics, double diffusion, diffusion coefficient
\end{abstract}

PACS: 89.75.Kd, 47.20.Bp, 47.54.-r, 47.70.Fw

\footnotetext{
${ }^{*}$ Corresponding author

Email addresses: horvathd@chem.u-szeged.hu (Dezső Horváth), atoth@chem.u-szeged.hu (Ágota Tóth) 


\section{Introduction}

Various types of spatiotemporal patterns surround us like corals in the ocean, clouds, the spirals in the galaxies.[1,2] In chemical systems, chemical gardens $[3,4,5,6]$ and the regular precipitation bands evolving through simple precipitation reactions called Liesegang structures[7, 8, 9, 10] are known for centuries. One of the most studied reactions yielding intriguing spatiotemporal structures is the Belousov-Zhabothinsky reaction where malonic acid is oxidized by bromate in an acidic medium.[11] A spatial excitation in this reaction may form target patterns, spirals, or in three-dimensional media even scroll waves.[12, 13, 14, 15, 16] Stationary spatial patterns may also arise as a result of the difference in diffusion coefficients, like the striped or hexagonal Turing patterns.[17, 18, 19]

One of the simplest spatiotemporal patterns is the chemical front which develops from the coupling of an autocatalytic reaction with a transport process.[20, 21, 22, 23] If a planar front propagates into a homogeneous reactant mixture, it generally maintains its symmetry. Under certain circumstances, however, the front may lose stability in the direction perpendicular to the front propagation and a cellular structure evolves. The phenomenon is termed lateral instability. If the transport process associated with the chemical front is purely diffusion, the difference in the flux of the reactant and the autocatalyst product can induce the loss of symmetry.[24, 25] There are two conditions which have to be valid in order for a simple autocatalytic front to exhibit diffusive instability: the autocatalysis has to be strong (i.e., the front has to be a "pushed type" [26]) and the flux of the reactant towards the front has to be greater than that of the product.[24, 25] The first 
cellular structure with small amplitude was presented experimentally in the iodate-arseneous acid reaction when the autocatalyst iodide was bound by cyclodextrine.[27] The cells were more pronounced when hydrogen ions were bound to carboxylate containing hydrogels in the acid-catalyzed reaction of the chlorite oxidation of tetrathionate in a slight chlorite excess.[28, 29, 30] In all cases, the wavelength of the first appearing pattern was in the cm range and appeared in the order of hours. Although in the first experimental examples the necessary difference in diffusion was achieved by reversible immobile complex formation, it was also shown that this can be in principle achieved by removing the autocatalyst irreversibly,[31] or even using constant external electric field.[32, 33]

Lateral instability can also be induced when there is no difference in the diffusion coefficients but the density changes during the reaction. In this case, buoyancy is the major driving force and the instability is called convective instability. $[1,34]$ The pattern formation was characterized by dispersion curves experimentally for the first time by Böckmann and Müller[35] in 2000 in the iodate-arseneous acid (IAA) reaction system. The density decreases during the reaction, therefore the downward propagating fronts are stable, while the upward propagating ones give rise to cellular patterns with an approximately $3 \mathrm{~mm}$ average wavelength. At the same time the results were compared by various theoretical models.[36, 37] Yang et al.[38] have discussed the stability in terms of the so-called Damköhler number which can characterize the density fingering in an autocatalytic reaction front in porous medium. For a Hele-Shaw cell the Damköhler number is defined as

$$
D a=\frac{144 D \nu^{2} k^{\prime}}{\left(\Delta \rho g \cos \theta a^{2}\right)^{2}},
$$


where $D$ is the diffusion coefficient of the reactant, $\nu$ is the kinematic viscosity, $k^{\prime}$ is a pseudo-first order reaction coefficient, $\Delta \rho=\left(\rho_{p}-\rho_{r}\right) / \rho_{0}$ is the relative density change with $\rho_{p}, \rho_{r}$, and $\rho_{0}$ as the density of the product, the reactant, and the water, in turn. The orientation is given by $\theta$ which is the angle from the vertical, $g$ is the gravitational constant, and $a$ is the gap width of the cell. The decrease in Damköhler number enlarges the region of convective instability thus the patterns evolve on a faster time scale and their average wavelength decreases for the ascending IAA front.[39]

In a vertical slab, horizontally propagating planar fronts are also unstable when the density either decreases[40, 41, 42, 43] or increases[44] in the course of the reaction giving rise to a gravity current.

The pattern formation in the chlorite-tetrathionate $(\mathrm{CT})$ reaction where the kinetics of the reaction is different and the density increases throughout the reaction (just opposite to the IAA reaction) was also investigated quantitatively both experimentally[45] and theoretically.[38] In this system the downward propagating fronts are hydrodynamically unstable, while in thin solutions the upward propagating fronts are stable and retain their initial planar geometry. The effects of various physical parameters like the orientation[45] or the gap width of the cell were investigated in detail.[46] The change in the density[47] or the effect of solution viscosity[48] was also discussed in the highly exothermic CT reaction.

The resultant patterns in all cases appear very similar to that in the diffusive case but there is a significant difference in the length scale and the time scale of the patterns. In aqueous solutions of both the IAA and the $\mathrm{CT}$ reactions, the convective patterns that are of a $\mathrm{mm}$ scale evolve in a few 
seconds, which is two magnitude faster than the diffusive one, independently of the chemical kinetics.

The CT reaction has also served as an example case to show the existence of migration driven instability. We have shown both theoretically and experimentally that the appropriate external electric field may either induce cellular pattern formation or hinder the loss of stability.[49, 50] The former is exhibited when the electric field enhances the mixing of the reactant and the autocatalyst, while the opposite orientation may even lead to the complete extinction of reaction front in which case simple electrophoretic fronts arise. In this system, the length scale is in the range of that in the diffusion-driven scenario, the time scale, however, is significantly shorter. Again, the front is required to be a pushed type in order to exhibit migration driven instability.

It has been shown by De Wit, Merkin and co-workers that the coupling of the diffusive and convective cases yields new patterns. [51, 52, 53] The difference in the diffusion coefficients may give rise to convective instability in the otherwise stable system. Our long time goal is to find the appropriate experimental conditions for studying the interaction between the diffusive and the convective instability in the CT reaction, for which we have utilized the previously successful immobile binding to a polymer. This time the carboxylate groups are incorporated in a linear polymer instead of a cross-linked matrix to allow macroscopic fluid motion. The first step has to be the determination of the onset of the diffusive instability in horizontally propagating fronts in which case convection can be negligible. We were, however, startled by a new type of cellular pattern arising under special experimental conditions. The cellular fronts observed are definitely not diffusively induced patterns 
and presumably are the result of convection taking place at a reaction front propagating horizontally in a thin layer of solution. In this manuscript we are going to show that a unique cellular convective structure can evolve due to the difference in diffusion of the reactant and the autocatalyst.

\section{Experimental}

Reagent-grade chemicals (Sigma, Aldrich, Reanal) of the CT reaction were used except for $\mathrm{NaClO}_{2}$, which was recrystallized twice as described earlier[54] to reach at least $95 \%$ purity. Polyacrylic acid with a $100 \mathrm{kDa}$ typical molecular weight (Aldrich) was used to prepare the sodium polyacrylate solution which can bind the hydrogen ion produced in the course of the reaction. A $0.30 \mathrm{M}$ stock solution was prepared by measuring out sodium hydroxide and polyacrylic acid solution in 1:1 ratio. The solutions, with compositions summarized in Table 1, were then prepared at room temperature and injected into a $16 \mathrm{~cm}$ wide, $0.4 \mathrm{~mm}$ or $0.8 \mathrm{~mm}$ thick, and $12 \mathrm{~cm}$ long reaction vessel with two $8 \mathrm{~mm}$ thick Plexiglas walls as shown in Fig. 1. The cell was positioned horizontally and planar fronts were initiated electrochemically by applying a $2.8 \mathrm{~V}$ potential difference between two Pt wires (0.25 $\mathrm{mm}$ in diameter) for $30 \mathrm{~s}$. We monitored the traveling fronts through an

appropriate cut-off filter - to enhance the contrast between the reacted and the fresh solutions - by a monochrome CCD camera, and frames of $768 \times 576$ pixels were digitized in $5 \mathrm{~s}$ intervals.

From the front position, given as the point of inflection in the gray scale values along the direction of propagation ( $x$ coordinate), the mean front position was determined by averaging it perpendicular to the direction of the 
propagation ( $y$ coordinate). In order to characterize the evolution of front profiles, dispersion curves - defined as the growth rate as a function of the wave number-were constructed. One-dimensional Fourier transformation was applied on the front profiles with a Hann-window to correct for the finite size of the observation window. From the time evolution of the Fourier amplitudes the growth rates were calculated for each mode as the slope of the linear regime as described in detail previously.[45] The cellular patterns observed are characterized by calculating the spatial autocorrelation according to[55]

$$
R_{j}=\frac{\sum_{i=1}^{N-j}\left(x_{i}-\bar{x}\right)\left(x_{i+j}-\bar{x}\right)}{\sum_{i=1}^{N}\left(x_{i}-\bar{x}\right)^{2}}
$$

where $x_{i}$ is the front position, $\bar{x}$ is the average front position and $N$ is the number of points perpendicular to the direction of propagation. Knowing the spatial resolution $\Delta y, R_{j}$ is converted to its dimensional form as $R_{y}=\Delta y R_{j}$.

The density of the reactant and the product solution was measured by an AP Paar DMA 58 digital density meter within $10^{-5} \mathrm{~g} / \mathrm{cm}^{3}$ precision. The viscosity of the same solution was obtained by Ostwald viscometry.

In order to determine the diffusion coefficients, we prepared $2.4 \mathrm{ml}$ solution with composition in Table 1 where the concentration of sodium polyacrylate was $25.0 \mathrm{mM}$ and sodium chlorite was substituted by sodium acetate. For the NMR measurements, deuterated water (Aldrich) in $10 \%$ amount was included in the solution necessary for locking the magnetic field. The diffusion coefficients were determined by pulse-field gradient spin-echo NMR using a Bruker Avance DRX 500 spectrometer. The combination of bipo- 
lar pulse pair longitudinal eddy-current delay (BPPLED) sequence and WATERGATE sequence was used, the latter applied for the suppression of water signal. Rectangular pulses of $0-31 \mathrm{G} / \mathrm{cm}$ gradients separated by $\Delta=200 \mathrm{~ms}$ diffusion time were employed. The duration of gradient pulses was set to $\delta=$ $2 \mathrm{~ms}$ to determine the diffusion coefficient of sodium acetate and to $\delta=4 \mathrm{~ms}$ for the polymer. All measurements were carried out using NMR tubes with 5 $\mathrm{mm}$ diameter. The spectra were processed by the GIFA program package[56] and the experimental data were evaluated with home-written codes.

\section{Results and Discussion}

The propagation of planar fronts in horizontal direction is first monitored in $0.4 \mathrm{~mm}$ thin solution layers. In solutions containing $22.5 \mathrm{mM}$ sodium polyacrylate, $75 \%$ of the hydrogen ion produced during the reaction is bound to the polymer. Under these conditions the horizontally propagating planar front is stable and it retains its geometry inherited from the initiation as illustrated in Fig. 2(a-c). On further increasing the concentration of the sodium polyacrylate we find the onset of diffusive instability at $27.5 \mathrm{mM}$. At higher sodium polyacrylate concentrations, i.e., within the region of diffusive instability, the planar front loses stability, yielding a cellular structure like in Fig. 2(d). The cells with slightly curved leading segments are joined in sharp cusps and the average wavelength is in the order of $\mathrm{cm}$, similarly to the previously observed patterns in hydrogels containing methacrylate polymerized into the matrix in order to bind hydrogen ion.[29] The further increase to $30 \mathrm{mM}$ will only increase the amplitude of the patterns without significantly affecting the wavelength. At these geometrical conditions, convection has a 
significant effect on the horizontal reaction fronts only if the solution layer is positioned vertical and planar fronts are propagating either upward or downward, similarly to the cases in our previous work.[47] In this very thin horizontal layer, hydrodynamics may become important because the front speed drastically decreases upon the addition of a polymer that reversibly binds the autocatalyst produced in the course of the reaction. Even though the density change is small and heat effects are negligible, the significant increase in the chemical time scale due to the reversible binding decreases the Damköhler number so much that convection on the shorter time scale can alter the initial planar front geometry.

Upon a slight increase in the height of the solution layer, however, cellular patterns shown in Fig. 3 evolve unexpectedly in a parameter range below the onset of diffusive instability suggesting that convection may be the major pattern forming process. In order to understand the phenomenon, we have carried out a study where we systematically vary the amount of hydrogen ion bound by the sodium polyacrylate via increasing the concentration of the macromolecule. Interestingly, we have found that there is a narrow range of polymer concentration within which a cellular pattern evolves from the initial planar structure. The cellular pattern is very regular but has a significantly shorter length scale than the diffusion-driven cellular front of Fig. 2(d), as shown by their autocorrelation function in Fig. 4. Beyond the narrow range but below the onset of diffusive instability, we find again that planar reaction fronts retain their planar symmetry, as shown in Fig. 3(c).

The stability of a planar front is best characterized by the construction of the dispersion curves. For a certain chemical composition, they are obtained 
from the front profiles during the initial development (see Figs. 5 and 6). For the system in the thinner solution layer, the planar geometry remains as illustrated in Fig. 2(b) and all spatial modes in the corresponding dispersion curve have negative growth rates as shown in Fig. 7. Thus, the planar front is stable (cf. Fig. 2(b)). At slightly greater thickness, a cellular structure evolves from the planar front as the modes with $k<6.8 \mathrm{~mm}^{-1}$ grow exponentially. The cellular pattern observed initially is dominated by the fastest growing mode of $k=4.5 \mathrm{~mm}^{-1}$, yielding an average wavelength of $\lambda=1.4 \mathrm{~mm}$ in accordance with Fig. 3(b).

For a quantitative characterization of the reactant and the product solution responsible for this observed unexpected behavior, we have determined both their density and viscosity, summarized in Table 2. The solutal density change associated with the reaction exhibits only an insignificant decrease across the range where the cellular patterns evolve. The values $(2.5-2.1) \times 10^{-4} \mathrm{~g} / \mathrm{cm}^{3}$ fall in the same range as those observed for the classical experimental setups excluding the polymer solution.[45, 47] Although the reaction itself is exothermic, due to the effective heat transfer from the very thin layer of solution to the surrounding though the walls the temperature rise is less than $0.05 \mathrm{~K}$. The thermal component of the density change is therefore in the order of $10^{-5} \mathrm{~g} / \mathrm{cm}^{3}$ and hence may be considered negligible with respect to the solutal component. Because in the experimental system the product solution is denser than the reactant, the location of the propagating reaction front in the third dimension of the thin layer is expected to be tilted from the vertical. In our system the deviation of the front orientation from the vertical is drastically enhanced because the velocity of propagation 
is significantly decreased by the addition of the polymer binding the autocatalyst as shown in Table 3. The reaction front therefore needs significantly more time to fill the entire solution thickness in contrast to the classical systems in the absence of the polymer, where the front velocity is in the range of $70 \mathrm{~cm} / \mathrm{h}$.[45, 46] The extent of tilting is revealed by the gray scale images shown in Figs. 2 and 3, where a gradual change in the grayscale between the reactant and product is observed even though the interface at the reaction front is in fact sharp. The denser product has the expected tendency to advance on the bottom of the layer only at smaller polymer concentration; the scenario changes upon the increase in polymer content (presented in Figs. $2(\mathrm{c})$ and $3(\mathrm{c})$ ), the product now propagates ahead on the top of the layer. For a clearer picture, the side view of the front is recorded in a separate set of experiments in which the width of the solution layer is decreased to $1 \mathrm{~mm}$. In case of $0.4 \mathrm{~mm}$ height, only a slight tilting is observed (see Fig. 8(a,b)) in accordance with that seen in the wide layer: the product advances on the bottom at lower polymer concentration and on the top at higher concentration. Increasing the height to $0.8 \mathrm{~mm}$, the tilting follows the same trend but is more pronounced, as shown in Fig. 8(c,e). In the transition zone, however, no stable front is observed and the front geometry becomes more complicated as revealed by Fig. 8(d). This parameter range is associated with the lateral instability observed in the wide solution layer (cf. Fig. 3(b)).

These results imply that double diffusive convection arises in the system. Since the thermal contribution to the density change is negligible, the difference in diffusion rate may result in the nonmonotonous change in density in the course of the reaction, we have therefore obtained information on the 
diffusional relations present in the system.

The pulsed field gradient spin echo (PFGSE) NMR measurements allow the monitoring of molecular displacement even in reactive systems. [57, 58] Upon increasing the magnetic field gradient, the signal intensity $\left(I\left(g_{B}\right)\right)$ of a given species obeys the classical Stejskal-Tanner equation[59]

$$
I\left(g_{B}\right)=I(0) \exp \left[-\gamma^{2} \delta^{2} g_{B}^{2} D\left(\Delta-\frac{\delta}{3}-\frac{\tau}{2}\right)\right],
$$

where $\gamma$ is the gyromagnetic ratio of ${ }^{1} \mathrm{H}, \delta$ and $g_{B}$ are the strength and length of the gradient pulse, respectively with $\tau$ being the delay between the $90^{\circ}$ and $180^{\circ}$ pulses in the bipolar pulse pair sequence, $D$ is the diffusion constant of the species associated with the signal, and $\Delta$ is the diffusion time between the two bipolar gradient pairs.

In our ${ }^{1} \mathrm{H}-\mathrm{NMR}$ measurements, acetate ion — or acetic acid in acidic mediumacts as a probe species for small aqueous ions, besides which the indicator bromophenol blue and the polyacrylate are detectable. Their signal separates well in the one-dimensional spectrum as shown in Fig. 9, where acetate has a sharp peak at $1.83 \mathrm{ppm}$, while polyacrylate is identified by a pair of wide bands at 1.23-1.69 ppm and 1.90-2.16 ppm, respectively. Due to their separation, the intensity of each band is well described by a single exponential attenuation with increasing gradient strength as presented for acetic acid in Fig. 10. The diffusion coefficients obtained from the Stejskal-Tanner equation are listed in Table 4 for measurement in basic and acidic solution with $\mathrm{pH}$ corresponding to the reactant and product mixture, respectively. The significant difference between the diffusion coefficient of the probe species acetate and the linear polymer that binds the autocatalyst is also remarkable on the DOSY representation shown in Fig. 11. We also mention that the bromophe- 
nol blue indicator also has a small peak at $7.58 \mathrm{ppm}$, from the attenuation of which its diffusion coefficient can be determined. The larger error in the value for bromophenol blue indicator is associated with its smaller intensity.

Apart from showing that diffusion coefficients decrease with increasing molecular mass, Table 4 reveals that their difference between the basic and acidic solution is negligible even though the viscosity of the solution decreases by ca. $20 \%$. With respect to the double diffusive convection, therefore the faster diffusion rate of the autocatalyst hydrogen ion and its controlled binding to the linear polymer with negligible diffusion are significant, since there is no considerable difference between the diffusional relations in the reactant and product mixture.

\section{Conclusion}

In this work we have shown that the addition of a linear macromolecule in order to bind the autocatalyst in an acidic reaction front not only decreases the velocity of propagation and therefore facilitates convective motion in very thin solution layers but also introduces a controlled difference in the flux of species leading to double-diffusive convection. Although the product mixture is denser than the reactant throughout the applied parameter range, the increase in the concentration of polyacrylate leads to an inversion of the sign of the local density change at the front. As a result, in the transition zone at sufficient solution thickness - $0.8 \mathrm{~mm}$ in the present study-pattern formation transverse to the direction of propagation is observed. A detailed characterization of the system, including density and viscosity measurements, temperature monitoring, and the determination of diffusion coefficients by 
PFGSE-NMR spectroscopy, reveals that the cellular fronts arise because the apparent diffusion of the autocatalyst hydrogen ion is slower than that of the reactants with the flux of the former controlled by the amount of linear polymer applied. The observed cellular convective pattern having a shorter characteristic length scale is distinct from the lateral diffusive instability of the reaction front arising at higher polymer concentration in thinner layers. This transverse instability appears only when the height of the thin layer is not negligible, theoretical studies therefore need to construct three dimensional models for the description of this phenomenon.

\section{Acknowledgments}

The authors dedicate this paper to Professor Stefan C. Müller. Á. T. is especially indebted to him for his encouragement and continuous interest in her work.

This work was financially supported by the Hungarian Scientific Research Fund (OTKA K72365) and ESA (C98036). 


\section{References}

[1] Epstein, I. R.; Pojman, J. A. An Introduction to Nonlinear Dynamics: Oscillations, Waves, Patterns, and Chaos; Oxford University Press: Oxford, 1998.

[2] Gray, P.; Scott, S. K. Chemical oscillations and instabilities: Non-linear chemical kinetics; Oxford University Press: Oxford, 1990.

[3] Cartwright, J. H. E.; García-Ruiz, J. M.; Novella, M. L.; Otálora, F. J. Coll. and Interface Sci. 2002, 256, 351-359.

[4] Thouvenel-Romans, S.; Steinbock, O. J. Am. Chem. Soc. 2003, 125, $4338-4341$.

[5] Devon, R.; RoseFigura, J.; Douthat, D.; Kudenov, J.; Maselko, J. Chem. Commun. 2005, 13, 1678-1680.

[6] Pagano, J. J.; Bánsági, T. J.; Steinbock, O. J. Phys. Chem. C 2007, 111, 9324-9329.

[7] Müller, S. C.; Kai, S.; Ross, J. J. Phys. Chem. 1982, 86, 4078-4087.

[8] Müller, S. C.; Kai, S.; Ross, J. Science 1982, 216, 635-637.

[9] Sharbaugh III, A. H.; Sharbaugh Jr., A. H. J. Chem. Ed. 1989, 66, $589-594$.

[10] Zrínyi, M.; Gálfi, L.; Smidróczki, É.; Rácz, Z.; Horkay, F. J. Phys. Chem. 1991, 95, 1618-1620. 
[11] Field, R. J.; Burger, M. Oscillations and traveling waves in chemical systems; Wiley Interscience: New York, 1985.

[12] Zaikin, A. N.; Zhabotinsky, A. M. Nature (London) 1970, 225, 535-537.

[13] Ševčíková, H.; Marek, M. Physica D 1983, 9, 140-156.

[14] Ševčíková, H.; Marek, M.; Müller, S. Science 1992, 257, 951-954.

[15] Steinbock, O.; Schütze, J.; Müller, S. Phys. Rev. Lett. 1992, 68, 248251.

[16] Bánsági Jr., T.; Steinbock, O. Phys. Rev. Lett. 2006, 97, 198301.

[17] Turing, A. Philos. Trans. Roy. Soc. London B 1952, 237, 37-72.

[18] Castets, V.; Dulos, E.; Boissonade, J.; De Kepper, P. Phys. Rev. Lett. 1990, 64, 2953-2956.

[19] Ouyang, Q.; Swinney, H. L. Nature (London) 1991, 352, 610-612.

[20] Gribschaw, T. A.; Showalter, K.; Banville, D. L.; Epstein, I. R. J. Phys. Chem. 1981, 85, 2152-2155.

[21] Hanna, A.; Saul, A.; Showalter, K. J. Am. Chem. Soc. 1982, 104, $3838-3844$.

[22] Póta, G.; Lengyel, I.; Bazsa, G. J. Chem. Soc., Faraday Trans. 1989, $85,3871-3877$.

[23] Szirovicza, L.; Nagypál, I.; Boga, E. J. Am. Chem. Soc. 1989, 111, $2842-2845$. 
[24] Horváth, D.; Petrov, V.; Scott, S. K.; Showalter, K. J. Chem. Phys. 1993, 98, 6332-6343.

[25] Jakab, É.; Horváth, D.; Tóth, Á.; Merkin, J. H.; Scott, S. K. Chem. Phys. Lett. 2001, 342, 317-322.

[26] van Saarloos, W. Phys. Rep. 2003, 386, 29-222.

[27] Horváth, D.; Showalter, K. J. Chem. Phys. 1995, 102, 2471-2478.

[28] Tóth, Á.; Lagzi, I.; Horváth, D. J. Phys. Chem. 1996, 100, 1483714839.

[29] Horváth, D.; Tóth, Á. J. Chem. Phys. 1998, 108, 1447-1451.

[30] Fuentes, M.; Kuperman, M. N.; De Kepper, P. J. Phys. Chem. A 2001, 105, 6769-6774.

[31] Tóth, Á.; Horváth, D.; Jakab, É.; Merkin, J. H.; Scott, S. K. J. Chem. Phys. 2001, 114, 9947-9952.

[32] Horváth, D.; Tóth, Á.; Yoshikawa, K. J. Chem. Phys. 1999, 111, 10-13.

[33] Virányi, Z.; Szommer, A.; Tóth, Á.; Horváth, D. Phys. Chem. Chem. Phys. 2004, 6, 3396-3401.

[34] Pojman, J. A.; Epstein, I. R. J. Phys. Chem. 1990, 94, 4966-4972.

[35] Böckmann, M.; Müller, S. C. Phys. Rev. Lett. 2000, 85, 2506-2509.

[36] De Wit, A. Phys. Rev. Lett. 2001, 87, 054502. 
[37] Martin, J.; Rakotomalala, N.; Salin, D.; Böckmann, M.; Müller, S. C. J. Phys. IV. 2001, 11, 99-106.

[38] Yang, J.; D’Onofrio, A.; Kalliadasis, S.; De Wit, A. J. Chem. Phys. 2002, 117, 9395-9407.

[39] De Wit, A. Phys. Fluids 2004, 16, 163-175.

[40] Pojman, J.A.; Epstein, I.R.; McManus, T.J.; Showalter, K. J. Phys. Chem. 1991, 95, 1299-1306.

[41] Keresztessy, A.; Nagy, I.P.; Bazsa, Gy.; Pojman, J.A. J. Phys. Chem. 1995, 99, 5379-5384.

[42] Vasquez, D.A.; Littley, J.M.; Wilder, J.W.; Edwards, B.F. Phys. Rev. E 1994, 50, 280-284.

[43] Rongy, L.; Goyal, N.; Meiburg, E.; De Wit, A. J. Chem. Phys. 2007, $127,114710$.

[44] Schuszter, G.; Tóth, T.; Horváth, D.; Tóth, Á. Phys. Rev. E 2009, 79, 016216.

[45] Horváth, D.; Bánsági Jr., T.; Tóth, Á. J. Chem. Phys. 2002, 117, 4399-4401.

[46] Bánsági Jr., T.; Horváth, D.; Tóth, Á. Phys. Rev. E 2003, 68, 026303.

[47] Bánsági Jr., T.; Horváth, D.; Tóth, Á. Chem. Phys. Lett. 2004, 384, 153-156.

[48] Rica, T.; Horváth, D.; Tóth, Á. Chem. Phys. Lett. 2005, 408, 422-425. 
[49] Virányi, Z.; Horváth, D.; Tóth, Á. J. Phys. Chem. A 2006, 110, 36143618.

[50] Virányi, Z.; Tóth, Á.; Horváth, D. J. Eng. Math. 2007, 59, 229-238.

[51] D'Hernoncourt, J.; Merkin, J. H.; De Wit, A. Phys. Rev. E 2007, 76, $035301(\mathrm{R})$.

[52] D'Hernoncourt, J.; Merkin, J. H.; De Wit, A. J. Chem. Phys. 2009, 130, 114502.

[53] D'Hernoncourt, J.; Merkin, J. H.; De Wit, A. J. Chem. Phys. 2009, 130, 114503.

[54] Tóth, Á.; Horváth, D.; Siska, A. J. Chem. Soc., Faraday Trans. 1997, 93, 73-76.

[55] Tóth, T.; Horváth, D.; Tóth, Á. J. Chem. Phys. 2007, 127, 234506.

[56] Pons, J. L.; Malliavin, T. E.; Delsuc, M. A. J. Biomol. NMR 1996, 8, 445-452.

[57] Morris, K. F.; Johnson, C. S. J. Am. Chem. Soc. 1992, 114, 3139-3141.

[58] Ambrosone, L.; Ceglie, A.; Colafemmina, G.; Palazzo, G. Phys. Chem. Chem. Phys. 2002, 4, 3040-3047.

[59] Callaghan, P. T. Principles of nuclear magnetic resonance microscopy; Clarendon: Oxford, 1995. 
Table 1: Composition of the reactant solution.

\begin{tabular}{||l|c||}
\hline \hline$\left[\mathrm{K}_{2} \mathrm{~S}_{4} \mathrm{O}_{6}\right] / \mathrm{mM}$ & 5.00 \\
{$\left[\mathrm{NaClO}_{2}\right] / \mathrm{mM}$} & 20.00 \\
{$[\mathrm{NaOH}] / \mathrm{mM}$} & 1.00 \\
{$[$ Bromophenol blue $] / \mathrm{mM}$} & 0.16 \\
{$[$ Sodium polyacrylate $] / \mathrm{mM}$} & $22.5-26.2$ \\
\hline \hline
\end{tabular}


Table 2: Density and relative viscosity of solutions at various sodium polyacrylate concentrations.

\begin{tabular}{||c||c|c||c|c||}
\hline \hline \multirow{2}{*}{$\begin{array}{c}\text { [Sodium polyacrylate] } / \\
\mathrm{mM}\end{array}$} & \multicolumn{2}{|c||}{$\rho /\left(\mathrm{g} \mathrm{cm}^{-3}\right)$} & \multicolumn{2}{|c||}{$\eta / \eta_{\mathrm{H}_{2} \mathrm{O}}$} \\
\cline { 2 - 5 } & reactant & product & reactant & product \\
\hline \hline 22.5 & 1.00089 & 1.00114 & 1.30 & 1.05 \\
25.0 & 1.00102 & 1.00124 & 1.34 & 1.04 \\
26.2 & 1.00111 & 1.00132 & 1.33 & 1.05 \\
\hline \hline
\end{tabular}


Table 3: Front velocity with solutions of different sodium polyacrylate concentrations and solution thickness.

\begin{tabular}{|c|c|c|}
\hline \multirow{2}{*}{$\begin{array}{c}\text { [Sodium polyacrylate]/ } \\
\mathrm{mM}\end{array}$} & \multicolumn{2}{|c|}{$v /\left(\mathrm{cm} \mathrm{h}^{-1}\right)$} \\
\hline & thickness: $0.4 \mathrm{~mm}$ & thickness: $0.8 \mathrm{~mm}$ \\
\hline 22.5 & $8.36 \pm 0.01$ & $9.65 \pm 0.01$ \\
\hline 25.0 & $4.54 \pm 0.01$ & $4.72 \pm 0.01$ \\
\hline 26.2 & $3.20 \pm 0.01$ & $3.41 \pm 0.01$ \\
\hline
\end{tabular}


Table 4: Diffusion coefficients determined by PFGSE ${ }^{1} \mathrm{H}-\mathrm{NMR}$.

\begin{tabular}{||l||c|c||}
\hline \hline \multicolumn{1}{||c||}{} & \multicolumn{2}{c||}{$D /\left(\mathrm{cm}^{2} \mathrm{~s}^{-1}\right)$} \\
\cline { 2 - 3 } & basic solution & acidic solution \\
\hline \hline acetate/acetic acid & $(1.21 \pm 0.01) \times 10^{-5}$ & $(1.17 \pm 0.01) \times 10^{-5}$ \\
bromophenol blue & $(4.8 \pm 0.3) \times 10^{-6}$ & $(4.9 \pm 0.2) \times 10^{-6}$ \\
polyacrylate/polyacrylic acid & $(3.64 \pm 0.06) \times 10^{-7}$ & $(3.57 \pm 0.09) \times 10^{-7}$ \\
\hline \hline
\end{tabular}




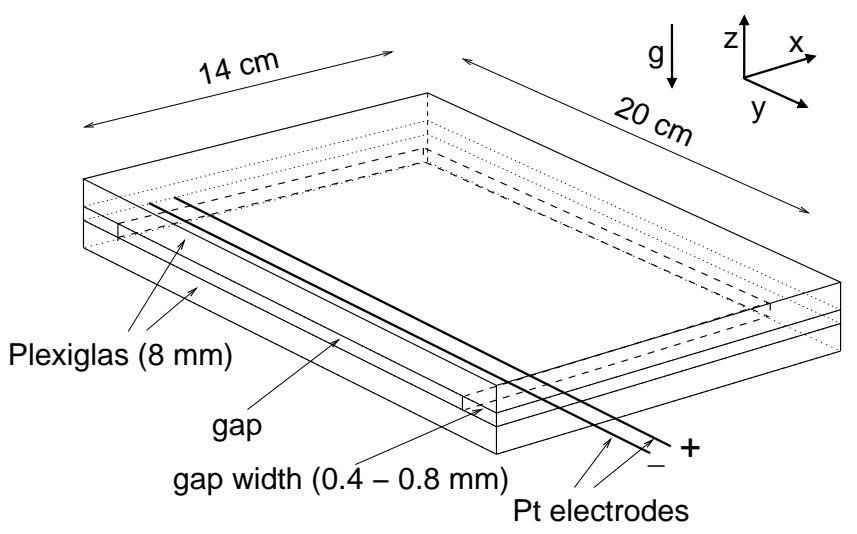

Figure 1: Scheme of the Hele-Shaw cell with the assignment of the axes.

T. Rica et al., submitted to Physica D 

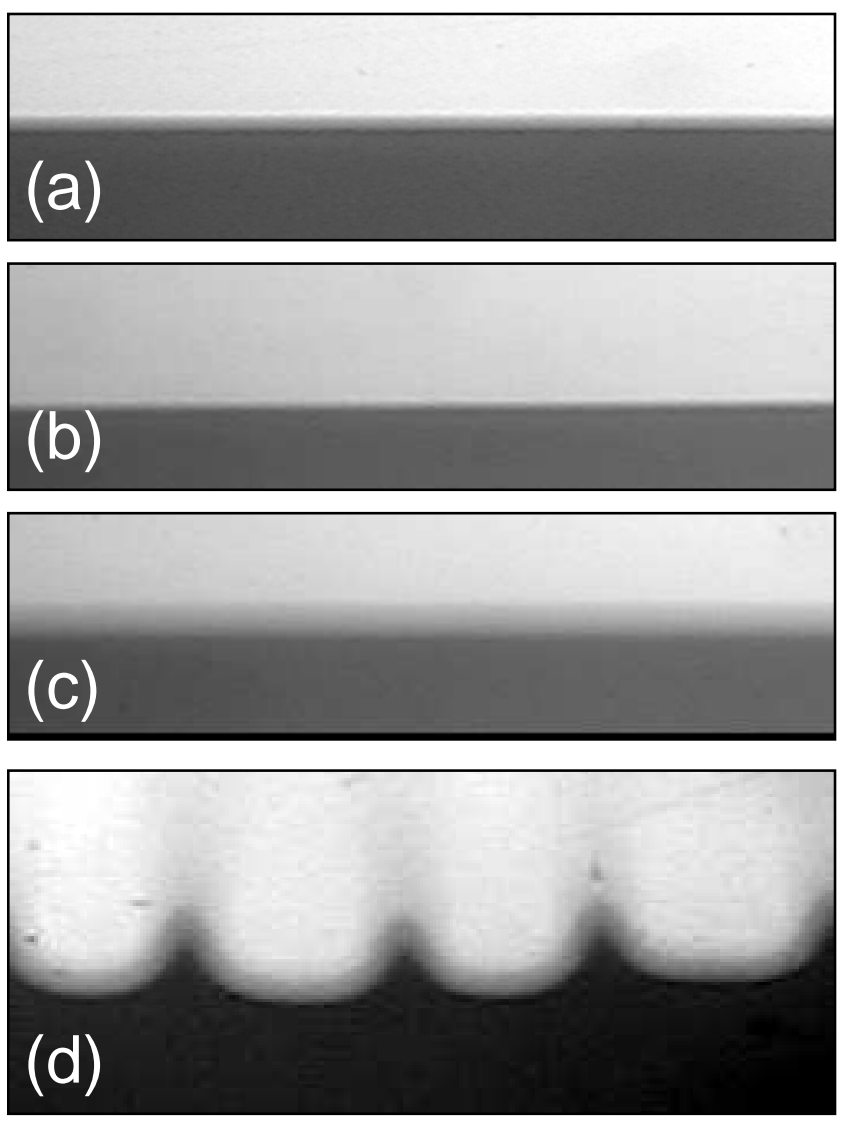

Figure 2: Images of patterns with various extent of hydrogen ion binding at $0.4 \mathrm{~mm}$ solution thickness. The concentration of sodium polyacrylate is (a) $22.5 \mathrm{mM}$ ), (b) 25.0 $\mathrm{mM},(\mathrm{c}) 26.2 \mathrm{mM}$, and (d) $30.0 \mathrm{mM}$. The dark region indicates the reactant solution, while the light one the product mixture. Field of view: $4.79 \mathrm{~mm} \times 1.32 \mathrm{~mm}$ for $(\mathrm{a}-\mathrm{c})$ and 17.8 $\mathrm{cm} \times 7.38 \mathrm{~cm}$ for $(\mathrm{d})$.

T. Rica et al., submitted to Physica D 

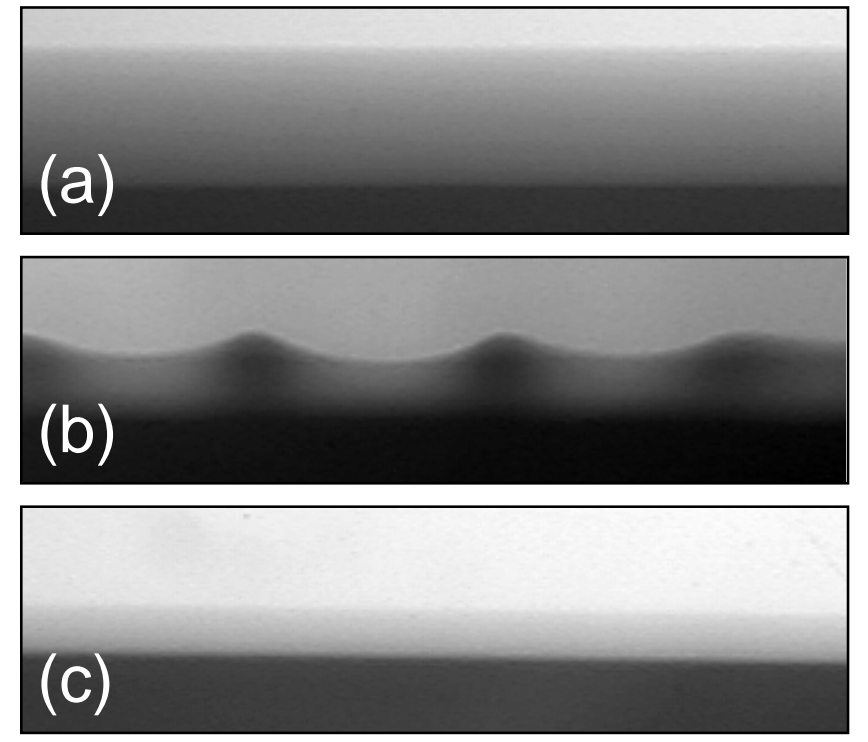

Figure 3: Images of patterns with various extent of hydrogen ion binding at $0.8 \mathrm{~mm}$ solution thickness. The concentration of sodium polyacrylate is (a) $22.5 \mathrm{mM}$, (b) 25.0 $\mathrm{mM}$, and (c) $26.2 \mathrm{mM}$. The dark region indicates the reactant solution, while the light one the product mixture. Field of view: $4.79 \mathrm{~mm} \times 1.32 \mathrm{~mm}$.

T. Rica et al., submitted to Physica D 


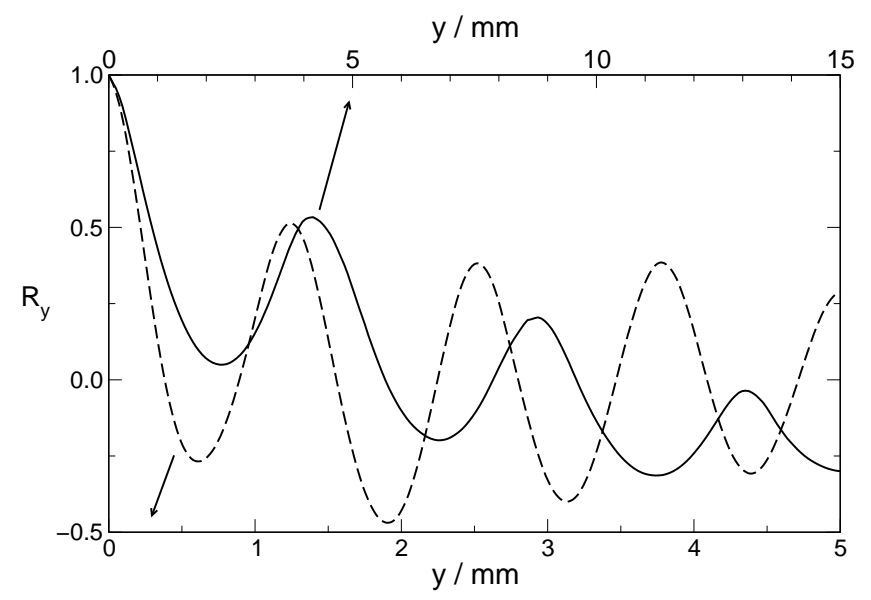

Figure 4: The spatial autocorrelation function $R_{y}$ for cellular patterns. The dashed line corresponds to the convection-driven (0.8 mm gapwidth, Fig. 3(b)) and the solid line to the diffusion-driven structure (0.4 mm gapwidth,Fig. 2(d)).

T. Rica et al., submitted to Physica D 


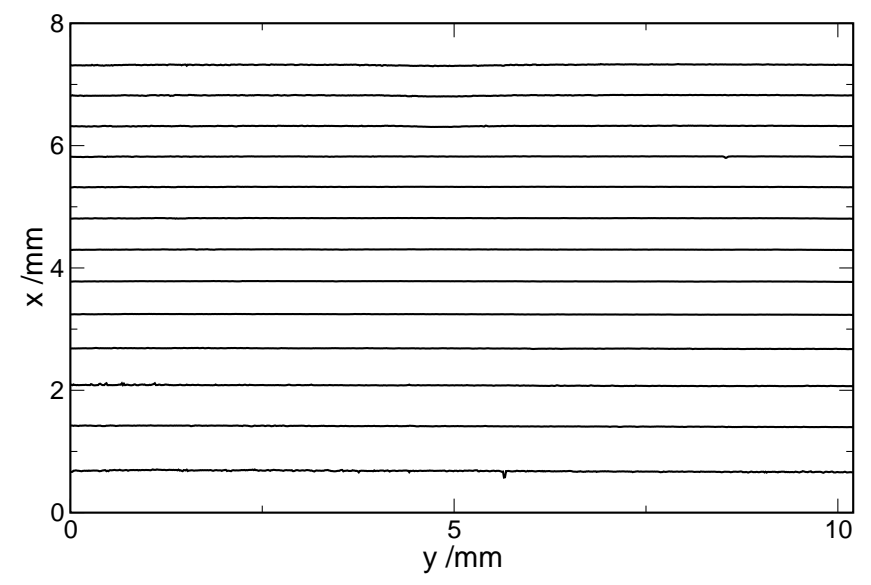

Figure 5: Front profiles showing the propagation of initially planar front at $\Delta t=40 \mathrm{~s}$ intervals with sodium polyacrylate concentration of $25 \mathrm{mM}$ and $0.4 \mathrm{~mm}$ solution thickness.

T. Rica et al., submitted to Physica D 


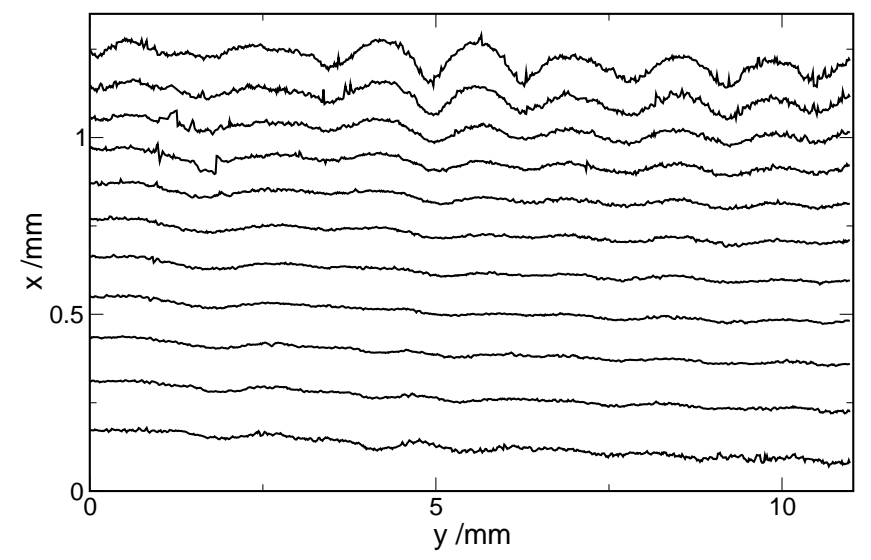

Figure 6: Front profiles showing the propagation of initially planar front at $\Delta t=10 \mathrm{~s}$ intervals with sodium polyacrylate concentration of $25 \mathrm{mM}$ and $0.8 \mathrm{~mm}$ solution thickness.

T. Rica et al., submitted to Physica D 


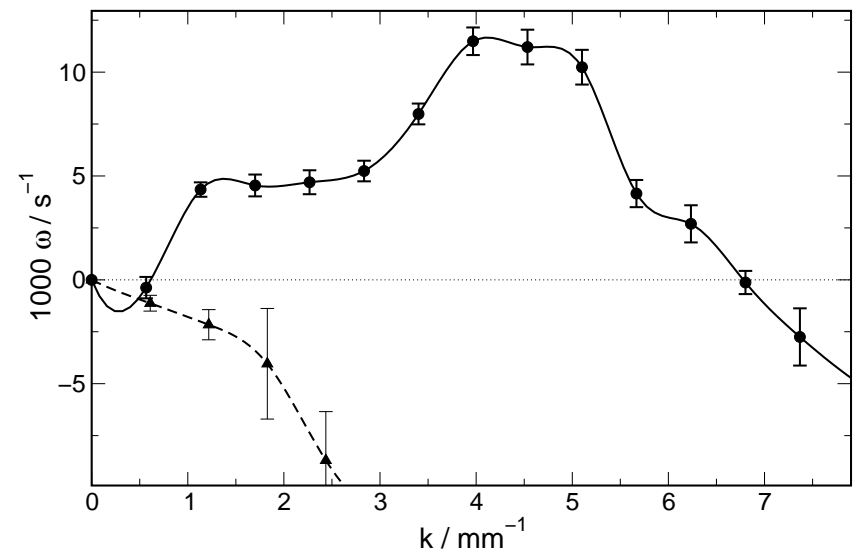

Figure 7: Dispersion curves for horizontally propagating fronts with sodium polyacrylate concentration of $25 \mathrm{mM}$ and $0.4 \mathrm{~mm}(\bullet)$ and $0.8 \mathrm{~mm}(\boldsymbol{\Lambda})$ gap width. The solid and the dashed lines are drawn to aid the eye.

T. Rica et al., submitted to Physica D 


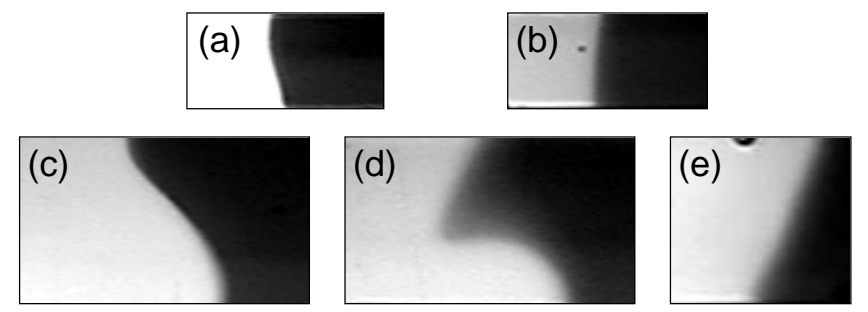

Figure 8: Side view images of reaction fronts with various extent of hydrogen ion binding. The concentration of sodium polyacrylate is (a) $22.5 \mathrm{mM}$, (b) $25.0 \mathrm{mM}$, at $0.4 \mathrm{~mm}$ solution thickness, and (c) $22.5 \mathrm{mM}$, (d) $25.0 \mathrm{mM}$, and (e) $26.2 \mathrm{mM}$ at $0.8 \mathrm{~mm}$ solution thickness. The dark region indicates the reactant solution, while the light one the product mixture.

T. Rica et al., submitted to Physica D 


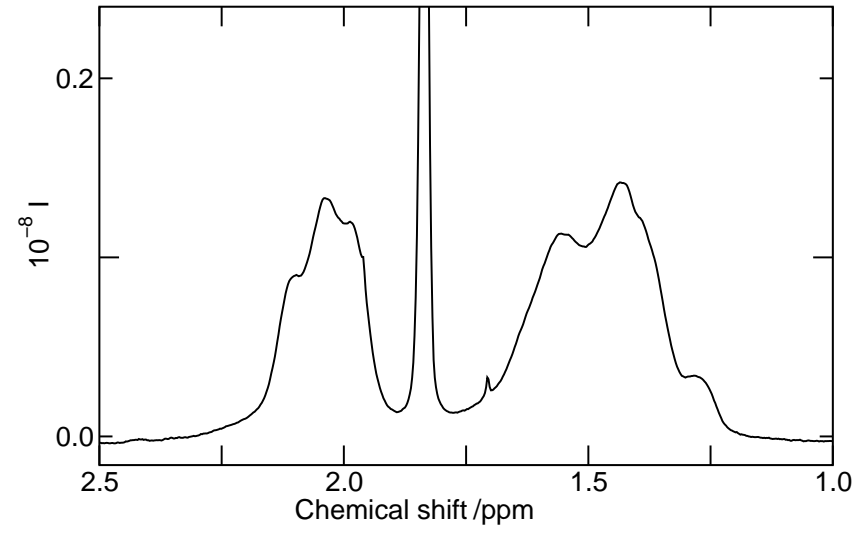

Figure 9: A one-dimensional ${ }^{1} \mathrm{H}-\mathrm{NMR}$ spectrum of the solution corresponding to the reactant mixture.

T. Rica et al., submitted to Physica D 


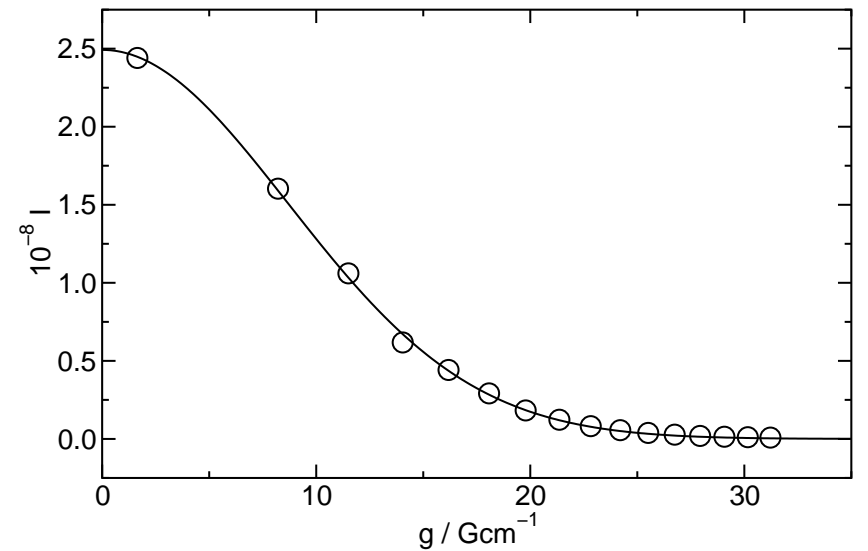

Figure 10: Signal intensity for acetic acid as a function of the gradient strength. The solid line is the fitting according to the Stejskal-Tanner equation.

T. Rica et al., submitted to Physica D 


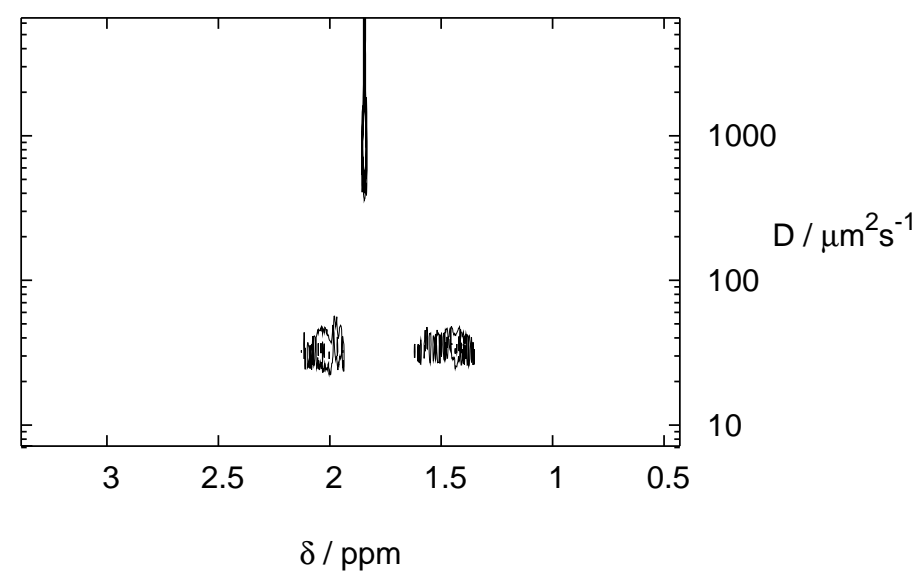

Figure 11: DOSY spectrum of the basic solution corresponding to the reactant mixture.

T. Rica et al., submitted to Physica D 